C-A/AP/\#268

February 2007

\title{
RHIC Run 7 Feedback Ramp Tunes
}

\author{
P. Cameron
}

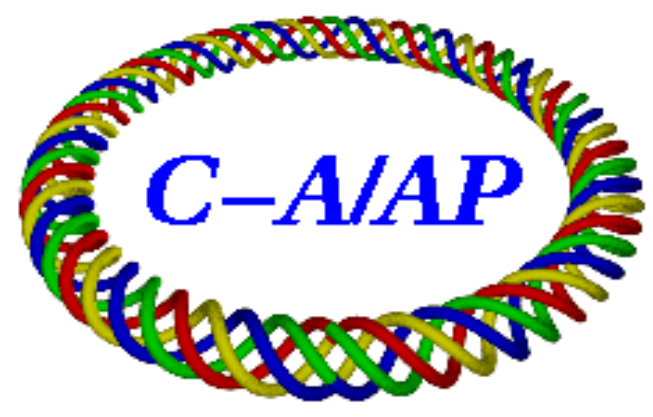

Collider-Accelerator Department Brookhaven National Laboratory Upton, NY 11973 


\title{
RHIC RUN 7 FEEDBACK RAMP TUNES
}

\author{
Peter Cameron, C-AD
}

\section{THE EFFECT OF MAINS HARM ONICS}

The RHIC baseband tune measurement system (BBQ) is extremely sensitive, capable of detecting beam motion of the order of $10 \mathrm{~nm}$. This sensitivity has revealed the presence of harmonics of the power line frequency in the betatron spectrum [1]. These harmonics are $\sim 30 \mathrm{~dB}$ above the BBQ noise floor at injection and store, and $\sim 70 \mathrm{~dB}$ above the noise floor during ramping. The effect of these harmonics must be considered in selecting tunes used for ramps that employ tune and coupling feedback. Two simple examples will give the reader some sense of how strongly these harmonics affect BBQ functioning.

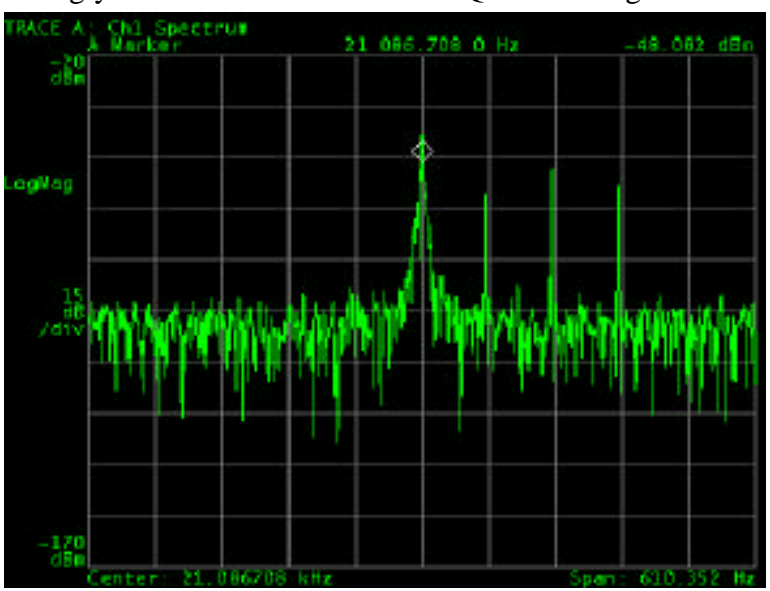

Figure 1: Simulation of mains harmonics

Figures 1 and 2 show data taken during bench testing in preparation for Run 7. In Figure 1, the relatively broad spectral line in the center of the figure results from the BBQ being locked to a test resonator. The three sharp lines to the right come from a signal generator used to simulate the effect of mains harmonics, the carrier being frequency modulated at $60 \mathrm{~Hz}$ to create the sidebands on either side. The carrier is $\sim 8 \mathrm{~dB}$ down from the BBQ excitation, and the sidebands are $\sim 15 \mathrm{~dB}$ down. For the measurements shown in Figures 1 and 2 the resonator was de-Q'd, so that the amplitude of its response to the signal generator remains relatively constant as the generator frequency is varied.

Figure 2 shows the interaction of the BBQ with these simulated mains harmonics as they are swept across the BBQ excitation. Time flows from top to bottom in this figure, with most recent time at the bottom. As the first sideband approaches it captures the BBQ, despite the fact that it is $15 \mathrm{~dB}$ down, and holds it for $\sim 30 \mathrm{~Hz}$ of sweep. During this time additional sidebands appear $\sim 10 \mathrm{~Hz}$ away from the $\mathrm{BBQ} /$ mains harmonic line, suggesting that the
BBQ is verging on instability. As the 'mains' carrier approaches the BBQ excitation it also appears to 'capture' the BBQ (in some sense of the word), but here it appears to be repelling rather than attracting. In frequency modulation the carrier and sidebands are 180 degrees out of phase. The observed difference in effect of the 'mains' carrier and sideband on the BBQ can probably be understood as a result of this phase shift. Again, the effect of the 'mains harmonic' on the BBQ is strong, despite the fact that the carrier is $8 \mathrm{~dB}$ down from the $\mathrm{BBQ}$.

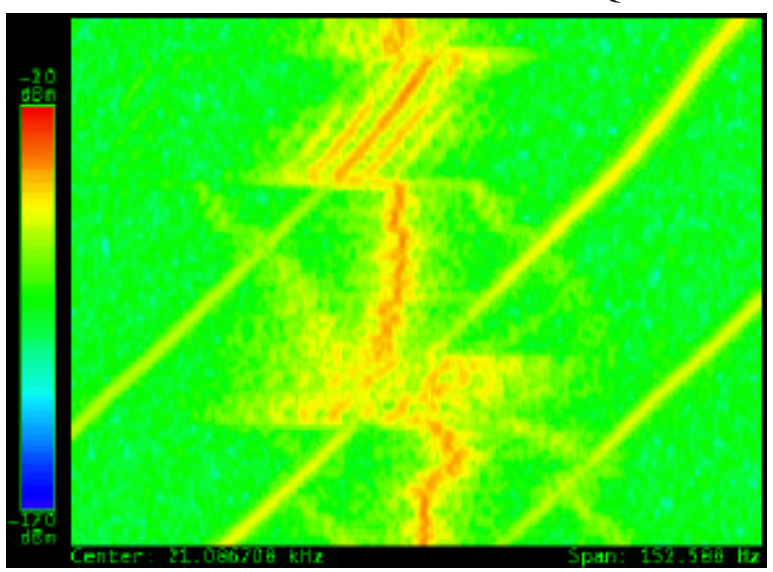

Figure 2: Walking the simulated mains harmonics across the $\mathrm{BBQ} /$ test resonator

Figure 3 shows dat a taken at store during Run 6. Again time flow s from top to bottom. At the top of the figure, the BBQ was locked to the betatron line. The BBQ excitation was such that its amplitude was $\sim 30 \mathrm{~dB}$ stronger than the weak mains harmonics, and $\sim 15 \mathrm{~dB}$ stronger than the strong mains harmonics. The phase of the lock was zeroed, then swept in increments of 10 degrees. The lock can be seen to jump discretely from one mains harmonic to the next until it is adjacent to a strong harmonic, where it remained pinned by the phase information of the strong harmonic for 50 degrees of phase change. It then jumped 6 harmonics to the next strong harmonic. Dialing the phase back towards zero, the BBQ again preferentially locked in the vicinity of mains harmonics while finding its way back to the starting point. It is useful to keep in mind that this data was taken at store, and that the mains harmonics are $\sim 40 \mathrm{~dB}$ stronger during ramping.

These examples hopefully make clear the strong effect of mains harmonics on the functioning of the BBQ, as well as the need to carefully select and control tunes during ramping. 


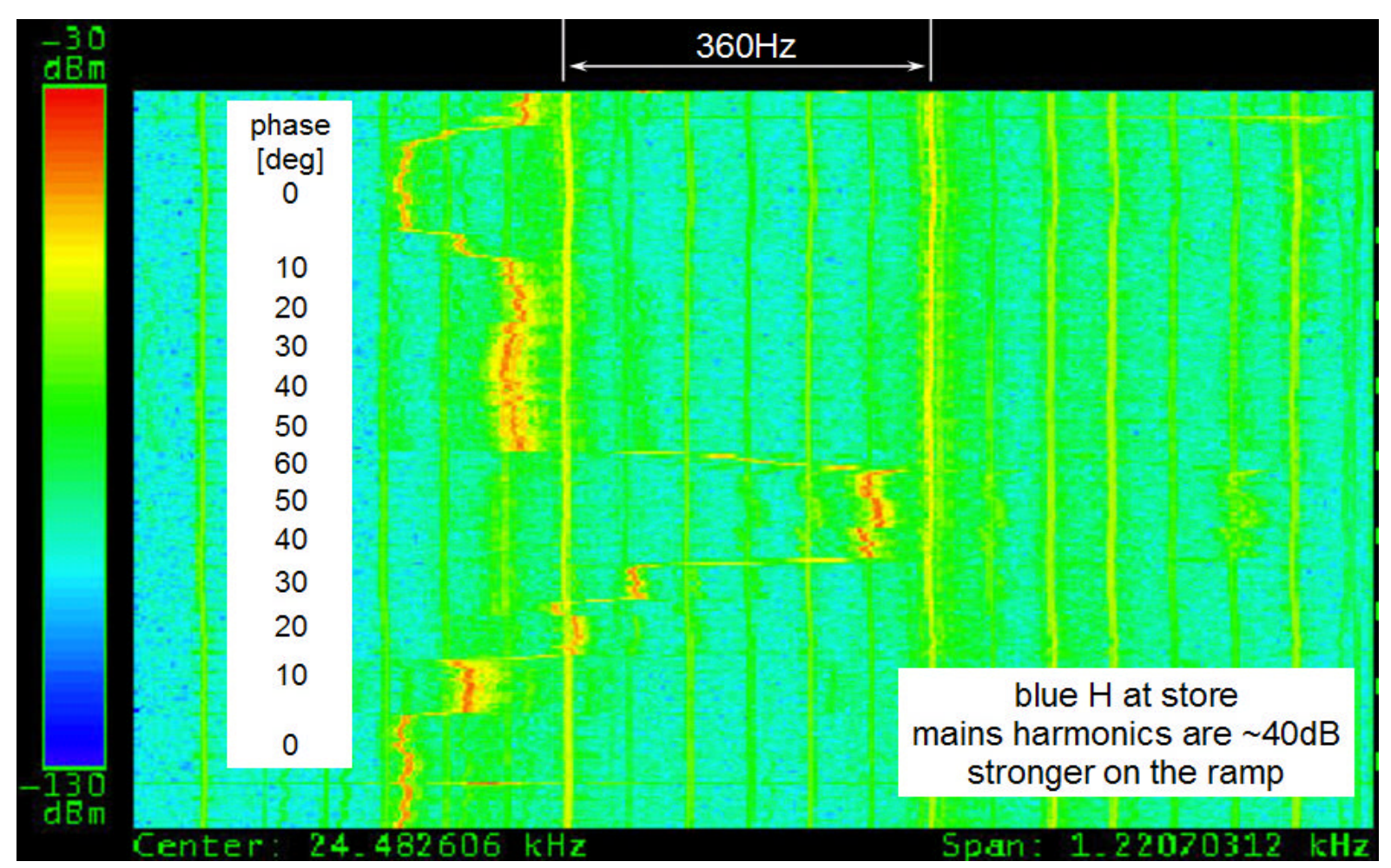

Figure 3: Effect of phase walk at store during Run 6

\section{DESIRED TUNES}

During Run 6 (with protons above transition at injection) the frequency swing up the acceleration ramp was small enough to permit closing the tune feedback loop through the ramp without pulling the tunes across mains harmonics. This is not the case for gold beam during Run 7. Frequency swing up the ramp for a horizontal tune of .230 a injection is shown in Figure 4. Rather than maintaining constant tunes during feedback ramps, it is desirable to maintain constant tune frequencies, to avoid pulling the tunes across the $\sim$ constant frequency mains harmonics. This can be accomplished by calculating desired ramp tunes from B $\rho$.

The nominal desired Run 7 tunes are .230, .215. Figure 5 shows the location of strong mains harmonics from Run 6. Due to the tunes used for proton running, data is available only down to a tune of $\sim .22$, which is above the desired vertical tune of $\sim .215$. However, experience has shown that mains harmonics are not strong in the vertical plane when RHIC is well decoupled, which should be the case during feedback ramps. Assuming that the same mains harmonics will be strong for Run 7 (not necessarily so, but probably the best guess), desired tunes at store for Run 7 are also shown in Figure 5. This note proposes that store tunes of $.22905, .21524$ be used for Run 7 feedback ramps.

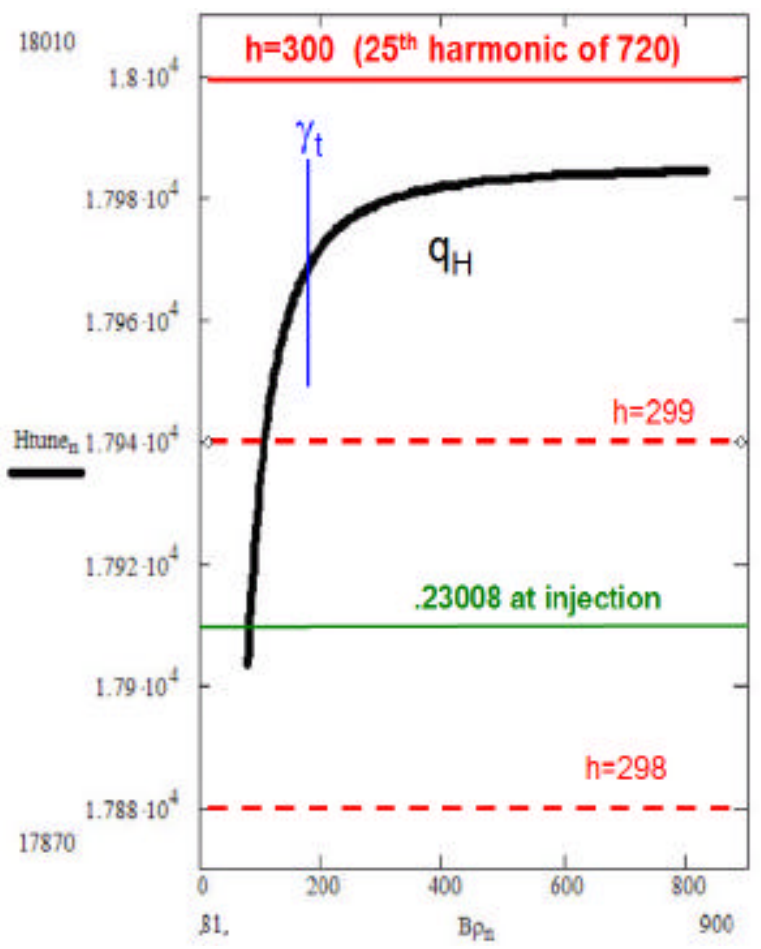

Figure 4: T une swing up the Au ramp 


\section{Blue horizontal mains harmonics on ramp 5 May 2006}

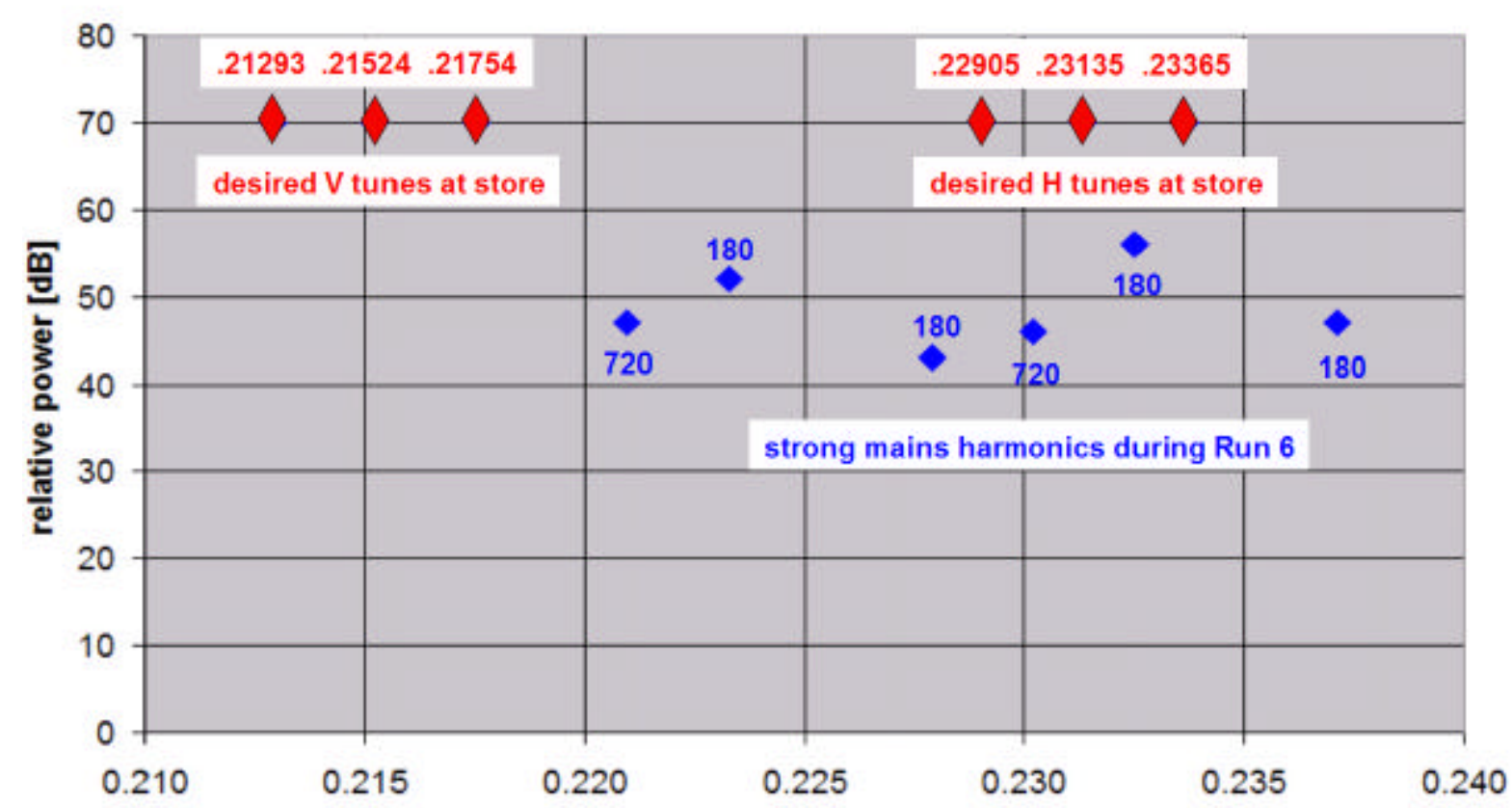

Figure 5: Strong mains harmonics during Run 6, and desired tunes for Run 7

\section{DESIRED TUNE CALCULATION}

Table 1 shows relevant machine and beam parameters [2] used for the desired tune calculation.

\begin{tabular}{|l|c|c|} 
parameter & symbol & value \\
Au charge $[\mathrm{q}]$ & $\mathrm{Q}$ & 79 \\
\hline Au mass $[\mathrm{GeV}]$ & $\mathrm{m}$ & 183.43312 \\
\hline RHIC radius $[\mathrm{m}]$ & $\mathrm{R}$ & 610.17538 \\
\hline Injection B $[\mathrm{T}-\mathrm{m}]$ & B $\rho$ inj & 81.11378 \\
\hline Store B $\rho[\mathrm{T}-\mathrm{m}]$ & B $\rho$ sto & 839.5 \\
\hline
\end{tabular}

Table 1: Machine and beam parameters

The maximum revolution frequency in RHIC is

$$
\text { Frev }_{\max }=\frac{c}{2 \pi R}
$$

or $78196.3 \mathrm{~Hz}$. For a given $\mathrm{B} \rho$, the revolution frequency [3] is

$$
\text { Frev }=\text { Frev }_{\max } \sqrt{1-\frac{1}{\left(\frac{Q \cdot B \rho \cdot c}{m}\right)^{2}+1}}
$$

which gives revolution frequencies of $77842.2 \mathrm{~Hz}$ at injection and $78193 \mathrm{~Hz}$ at store. To maintain constant tune frequencies, the desired tunes along the ramp are then

$$
\mathrm{v}_{\text {ramp }}=\frac{\mathrm{v}_{\text {store }} \cdot \text { Frev }_{\text {store }}}{\text { Frev }}
$$

which for the $.22905, .21524$ store tunes (shown below on Waldo's resonance diagram) gives

$$
v_{\text {rampH }}=\frac{17910 \mathrm{~Hz}}{\text { Frev }} \quad v_{\text {ramp }}=\frac{16830 \mathrm{~Hz}}{\text { Frev }}
$$

where Frev is calculated as shown in eqn (1). Injection tunes would then be $.23008, .21621$.

\section{ACKNOWLEDGEMENTS}

Thanks to Al Dellapenna for setting up and taking the data shown in Figures 1 and 2, and to Larry Hoff for a helpful conversation regarding referencing tunes to the revolution frequency at store, rather than injection.

\section{REFERENCES}

[1] P. Cameron et al, "Observations of Direct Excitation of the Betatron Spectrum by Mains Harmonics in RHIC", GAD AP Note 253, September 2006, available at http://www.rhichome.bnl.gov/AP/ap notes/cad ap index.html [2] RHIC Design Manual, available at http://www.rhichome.bnl.gov/NT-share/rhicdm/index.html [3] J. DeLong, private communication 


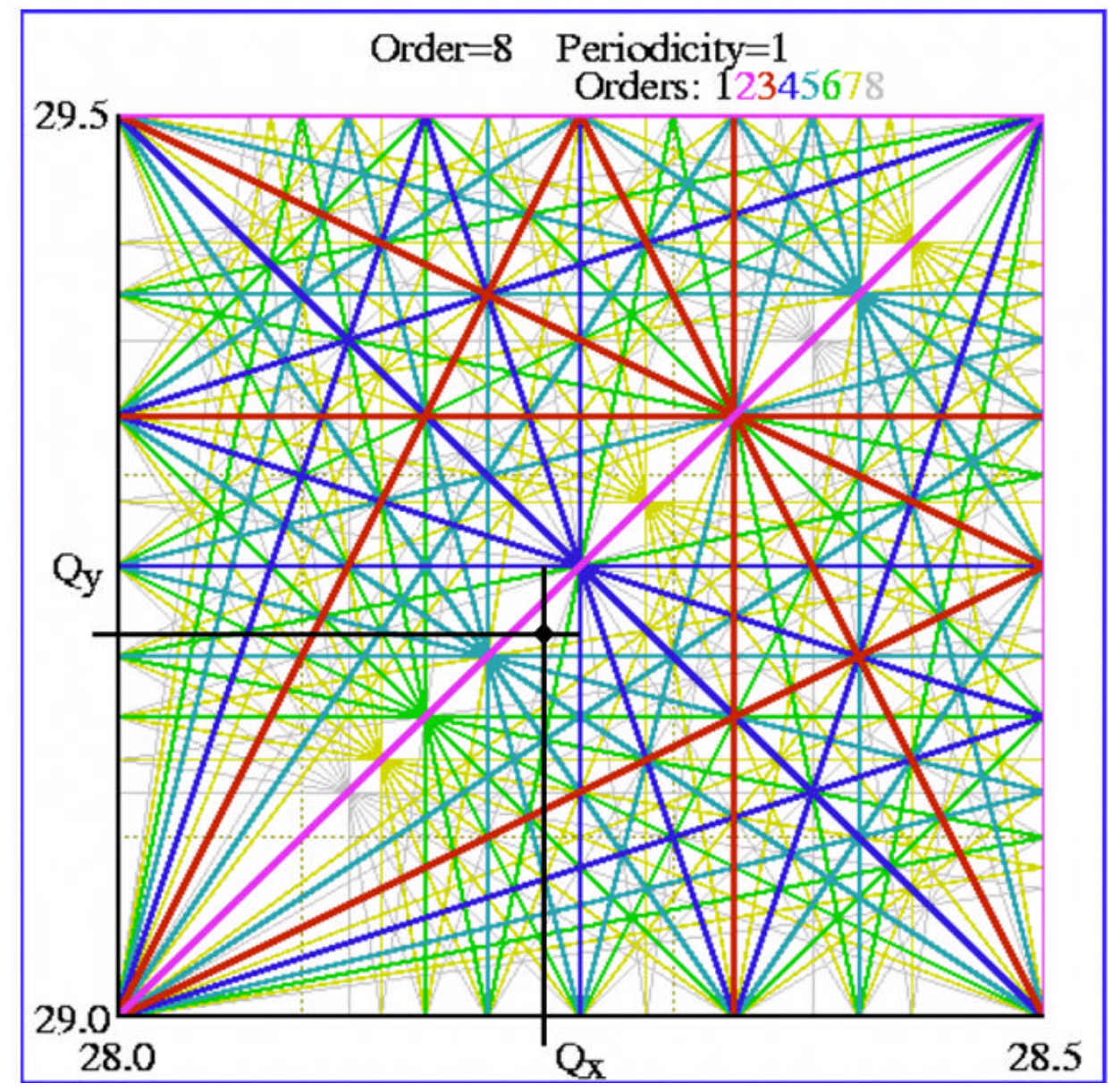

